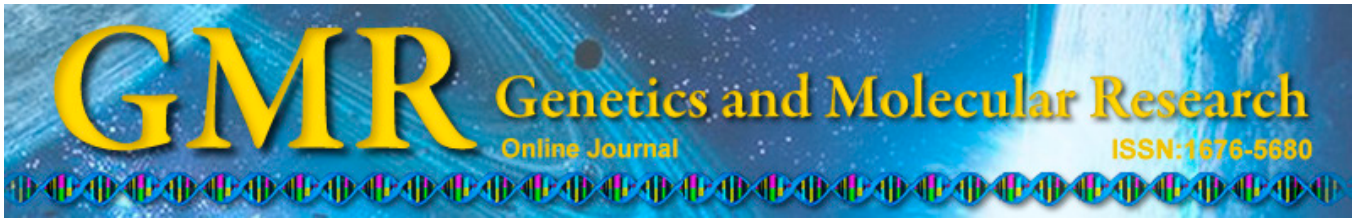

\title{
Characterization of abnormal epithelium after laser-assisted subepithelial keratectomy using in vivo confocal microscopy
}

\author{
J. Zuo ${ }^{1}$, C.W. Zhang ${ }^{2}$, X. Zhou ${ }^{2}$, W. Wei ${ }^{2}$ and Y.L. Wang ${ }^{2}$ \\ ${ }^{1}$ Department of First Clinical Medical College, \\ Nanjing University of Chinese Medicine, Nanjing, China \\ ${ }^{2}$ Department of Ophthalmology, \\ The Affiliated Hospital of Nanjing University of Chinese Medicine, \\ Nanjing, China \\ Corresponding author: C.W. Zhang \\ E-mail: chuanweizhang@yeah.net
}

Genet. Mol. Res. 14 (2): 4749-4756 (2015)

Received May 13, 2014

Accepted October 29, 2014

Published May 11, 2015

DOI http://dx.doi.org/10.4238/2015.May.11.7

\begin{abstract}
This study compared the abnormal corneal epithelium after laser-assisted subepithelial keratectomy (LASEK) with dystrophic cornea using in vivo using confocal microscopy (IVCM) and examined the effects of the abnormal epithelium on postoperative recovery of uncorrected distance visual acuity (UDVA) and sub-basal nerve plexus regeneration. The UDVA and wound healing were examined in 50 eyes of 25 patients undergoing LASEK at 1 week, 1 month, and 1 year postoperatively, including the visual acuity, slit lamp microscopy, and IVCM. After 1 week, the corneal epithelium was healed in all patients, but abnormal epithelial cells were detected in 33/50 patients using IVCM. Abnormal cells were limited to the surgical margin, and highly reflective granules were observed underneath. At 1 month and 1 year postoperatively, the abnormal epithelium was unchanged in size. At 1 year postoperatively, there were clear differences between the sub-basal nerve plexus in the normal and abnormal epithelium. At 1
\end{abstract}


month postoperatively, the UDVA was $>1.0$ in $88 \%$ of patients, which increased to $94 \%$ at 1 year, and there was no clear difference in the UDVA between abnormal $(\mathrm{N}=33)$ and normal $(\mathrm{N}=17)$ epithelium. After LASEK, abnormal epithelial cells may arise at the margin of the epithelial flap and persist 1 year postoperatively accompanied by poor regeneration of the sub-basal nerve plexus. However, this does not affect the UDVA postoperatively. The abnormal epithelium may be caused by residual necrotic basal cell debris on the epithelial basement membrane and abnormal neurotrophic metabolism between the corneal epithelium and nerve plexus.

Key words: Abnormal epithelium; LASEK; Confocal microscopy; Uncorrected distance visual acuity; Sub-basal nerve plexus

\section{INTRODUCTION}

During the first decade of the 21 st century, corneal refractive surgery started being performed on the order of femto-seconds. More patients have since chosen Femto-Second Lenticule Extraction or Small Incision Lenticule Extraction for myopia rectification. However, excimer laser corneal refractive surgery, the mainstay treatment for myopia and myopic astigmatism, is still widely used clinically. Recently, using live confocal microscopy, we retrospectively assessed patients who underwent Laser-Assisted in situ Keratomileusis (LASIK) and laser-assisted subepithelial keratectomy (LASEK) 10 years previously and found that in some patients who underwent LASEK, a corneal dystrophiclike abnormal epithelial region was present at the surgical margin. Thus, in the present study, we dynamically observed the early-stage regeneration of the cornea epithelium and sub-basal nerve plexus using in vivo confocal microscopy (IVCM) in LASEK patients, determined the time and localization of abnormal epithelial lesions, and assessed their effect on the sub-basal nerve plexus regeneration and the uncorrected distance visual acuity (UDVA) postoperatively.

\section{MATERIAL AND METHODS}

\section{Patients}

A total 50 eyes in 25 patients (10 men and 15 women) who underwent LASEK surgery at the First Affiliated Hospital of Nanjing University of Traditional Chinese Medicine were systematically examined from March 2012 to March 2013. The mean patient age was 23.8 years (range 20-32 years). The mean preoperative refraction in spherical equivalence was $-3.25 \mathrm{D}$ (range -1.75 to $-5.25 \mathrm{D}$ ) measured with an autorefractometer (Topcon RM-8800; Topcon America Corp, Paramus, NJ, USA). The mean preoperative pachymetry was $521 \mu \mathrm{m}$ (range 508-534 $\mu \mathrm{m}$ ) measured with an Orbscan II (Bausch \& Lomb).

A Technolas 217C (Bausch \& Lomb Chiron Technolas GmbH, Dornach, Germany) excimer laser was used for LASEK. The mean programmed diameter of the optical zone in all 50 eyes was $5.6 \mathrm{~mm}$ (range 5.5-6 $\mathrm{mm}$ ). The visual acuity, slit lamp microscopy, and IVCM were measured at 1 week, 1 month, and 1 year postoperatively included. 


\section{In vivo laser confocal microscopic observation}

All patients undergoing LASEK were examined by the same surgeon (Z.J.) in vivo using an HRT III/Rostock Cornea Module (HRT III/RCM; Heidelberg Engineering GmbH) confocal microscope 1 week, 1 month, and 1 year postoperatively. One drop of a local anesthetic $(0.4 \%$ benoxinate hydrochloride; Santen, Osaka, Japan) was instilled into the lower fornix of each eye. One drop of a polymer gel (Viscotears; CIBA Vision, Duluth, GA, USA) was applied onto the front surface of the microscope lens, and a Tomo-cap ${ }^{\circledR}$ (Heidelberg Engineering $\mathrm{GmbH}$ ) was mounted onto the holder to cover the microscope lens. The center and peripheral cornea were examined systematically layer by layer, and all corneal layer images were collected and saved.

\section{RESULTS}

\section{Postoperative in vivo confocal microscopy}

Abnormal epithelial regions were identified at the surgical margins 1 week postoperatively in 33 of 50 eyes. The most common findings were a highly reflective nucleus at the center of epithelial cells, indistinct cell borders, and fewer cell layers (Figure 1A). In addition, the lesions showed a granule-like highly reflective unidentifiable substance beneath the epithelial layers (Figure 1B); the area of each granule was slightly less than that of the basal cells, and the distance between the granules was larger than that of the basal cells.

The abnormal epithelium was map-like and found at the surgical margin. The lesions were uniformly sized, scattered randomly, and located at any position. At the 1-year follow-up, neither the number nor the abnormal epithelial cell area was changed.

At 1 week postoperatively, the sub-basal nerve plexus in the central and surrounding corneal surgical site was absent in all patients. However, 1 month postoperatively, a few subbasal nerve plexus located below the normal epithelium at the surgical margin showed growth into the cornea from the area outside the surgical site. The nerve structures were twisted, slender, and lacked branches.

At 1 year postoperatively, beneath the surgical margin of the normal epithelium, more sub-basal nerve plexus showed growth from outside of the surgical site towards the corneal center. They were twisted, slender, and showed a thinner diameter and increased tortuosity; moreover, the nerve structures showed some degree of branching. In contrast, below the abnormal epithelium, there was no sub-basal nerve plexus regeneration observed (Figure 1C). This change was not observed in the normal restored corneal epithelium and suborbital nerve fibers of the basement membrane (Figure 2A, B), but closely resembled the cornea of malnourished patients (Figure 3A, B, C). In addition, highly reflective granules were rarely observed (3/50) beneath the normal epithelium and regenerated sub-basal nerve plexus (Figure 4A, B).

\section{Postoperative uncorrected distance visual acuity}

At 1 month postoperatively, $88 \%$ of the eyes had a UVDA $>1.0$ (decimal), and at 1 year postoperatively, this increased to $94 \%$ (Figure 5). At 1 year postoperatively, there was no significant difference in the UDVA between the eyes with normal epithelium and those with abnormal epithelium (Figure 6). In the surgically treated eyes, 13 had a UDVA better than the 
preoperative UDVA 1 year postoperatively. Among them, 8 eyes had an abnormal epithelium, and 5 had a normal epithelium.
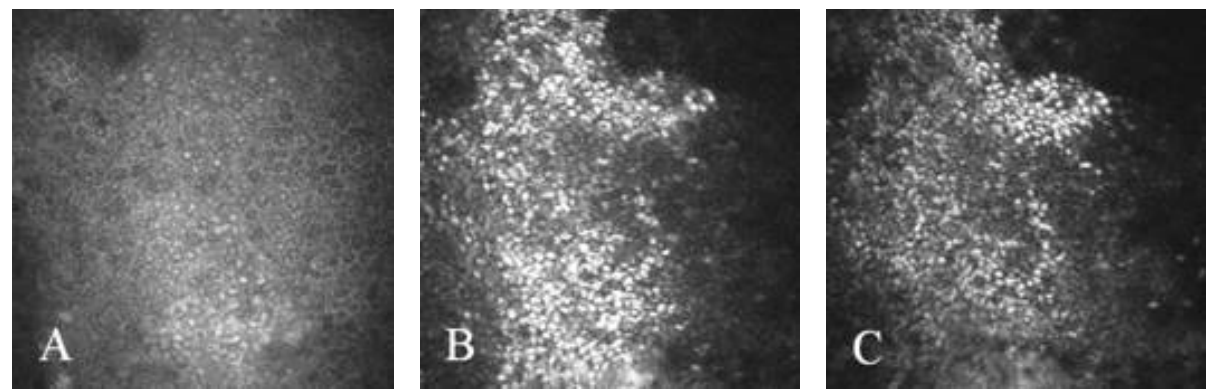

Figure 1. A. One week after surgery, abnormal epithelia region existed at the edge of corneal surgical area. In the center of epithelium cells, there were highly reflective nucleuses. The edge of the cells was blurred. Some cells had increased size. The number of cell layer reduced to only 2-4 layers. B. One week after operation, highly reflective uncertain materials were beneath abnormal epithelium cells. The size of each granule was slightly smaller than the basal cells. The distance between granules was larger than that of basal cells. C. Reparation of nerve fibers was not observed beneath abnormal epithelia one year after operation.
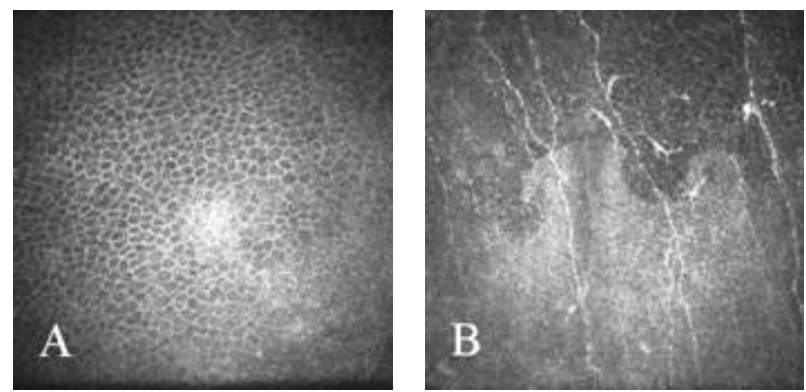

Figure 2. A. One week after operation, at the edge of corneal surgery area, the normal epithelia had normal size. Nucleus was not seen. The border was clear. There were 4-6 layers of cells. There were no highly reflective granules found on the basement membrane. B. One year after operation, sub-basal nerve plexus, which was in the area of normal epithelia on the edge of corneal surgery, recovered. The nerve grew from outside of surgical region into the center of cornea.
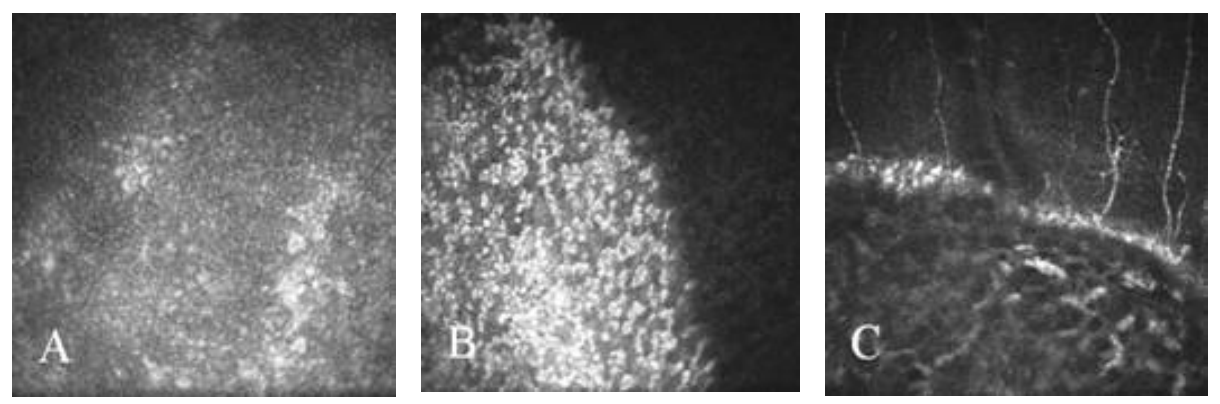

Figure 3. There were granule-like highly reflective materials in or beneath abnormal epithelia of 43-year old patient, who had basement membrane dystrophy. It accompanied with sub-basal nerve plexus damage. The morphology was similar to that of abnormal epithelia after LASEK. 

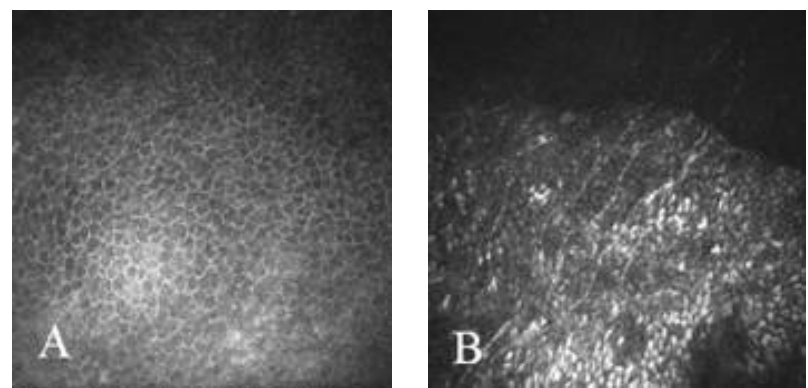

Figure 4. Sparsely-distributed highly reflective granule-like materials were observed beneath normal epithelia one year after operation. Below them, reparation of nerve fibers was seen.

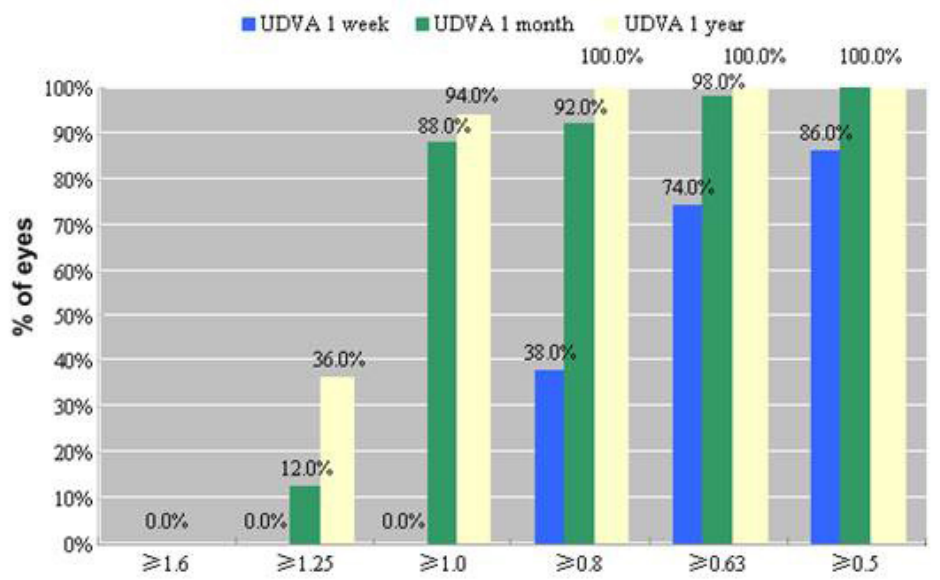

Figure 5. Postoperative uncorrected distance visual acuity at the 1-week, 1-month and 1-year visits.

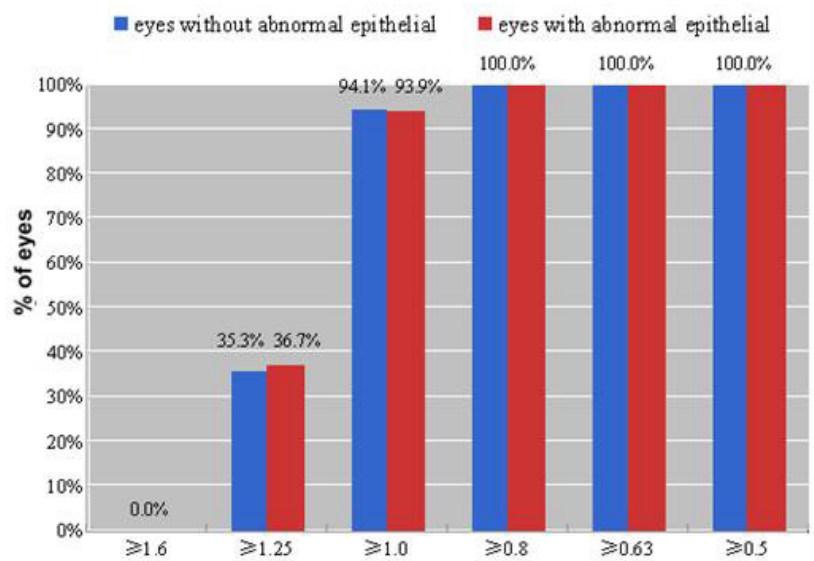

Figure 6. Postoperative uncorrected distance visual acuity of eyes without abnormal epithelium at the 1-year visit (clustered columns) versus postoperative uncorrected distance visual acuity of eyes with abnormal epithelium (stacked red line). 


\section{Postoperative slit lamp microscopy}

At 1 week postoperatively, all eyes with epithelial closure showed regeneration of epithelial cells. A total of 11 eyes developed grade 1 haze, and only 4 eyes developed grade- 2 haze at 1 month postoperatively. No eyes developed grade 3 or 4 haze. At the final visit, all of the treated eyes had a clear cornea.

\section{DISCUSSION}

Ever since the 1990s, LASEK has been used clinically as a safe, effective, and predictable method to treat myopia and myopic astigmatism (Taneri et al., 2004). The visual acuity after LASEK is similar to that following other procedures such as excimer laser corneal refractive surgery. Some studies have shown that the UDVA and visual quality of LASEK were even better than that of LASIK (Scerrati, 2001; Reilly et al., 2010; Bernátová and Synek, 2011). The flap, which is generated by soaking the tissue in alcohol, is either replaced by new epithelium $(120 \pm 5 \mathrm{~h})$ or is discarded intraoperatively (Taneri et al., 2011). The healthy new epithelium, like normal corneal epithelium, forms on the stromal bed of the surgical site, which prevents dislocation or loss of flap, epithelial ingrowth, and other complication seen in LASIK (Taneri et al., 2005; Rajan et al., 2005). In addition, regeneration of the sub-basal nerve plexus was significantly faster following LASEK than after LASIK (Lee et al., 2006). Biomechanical studies of the corneal stroma showed that placing the laser incision of the stromal layer in LASEK in the front caused minimal change in the corneal biomechanics and reduced the risk of thin corneal myopia postoperatively (Qazi et al., 2009).

Studies of complications after LASEK have focused on haze formation (Nakamura et al., 2001; Lin et al., 2004; Long et al., 2006; Thornton et al., 2007), but there are few reports describing epithelial complications. Using confocal microscopy, we observed the abnormal epithelial region after LASEK, which not only included the epithelial cell layer, but also the poor regeneration of the sub-basal nerve plexus. These abnormal changes were also observed by confocal microscopy in patients with corneal dystrophy. However, these changes had no effect on the postoperative visual recovery. Based on our observations on confocal microscopy and previous studies investigating the formation of abnormal epithelium, we inferred the following points:

The granule-like highly reflective unidentifiable debris beneath the abnormal epithelium on the surgical margin may be remnants of dead basal cells. Using electronic microscopy, Choi et al. (2008) observed that during LASEK, after the corneal epithelium was soaked in alcohol and scraped using an epithelial microhoe, less than $30 \%$ of the basal cells remained on the surface of basal membrane (Yulish et al., 2012). We inferred that the remaining basal cells in the central cornea were destroyed during the laser incision, and the surrounding basal cells were preserved because they were located outside of the laser incision. The remaining dead basal cells were covered by the transitional epithelial cells during regeneration of the corneal epithelium and thus were not dislodged. Once soaked in alcohol, the cells were difficult to degrade or absorb. Therefore, the necrotic debris resides long-term between the regenerated epithelial layer and the basal membrane. Previous pathology studies showed that alcoholsoaked stromal cells contained cytoplasmic debris and widened the cell gaps, and resembled the loosely formed, highly reflective granule-like material (Choi et al., 2008).

The remaining highly reflective granule-like material between the epithelial layer 
and basal membrane may cause dystrophy of the epithelial cells and sub-basal nerve plexus, and hinder basal membrane repair. Soluble nutrients continued to be exchanged between the epithelial cells and the sub-basal nerve plexus to maintain stability and promote regeneration including nerve growth factor, glial-derived neurotrophic factor, substance $\mathrm{P}$, and calcitonin gene-related peptide (You et al., 2000; Pallikaris et al., 2003; Huang et al., 2009; Chan et al., 2009; Kishi et al., 2010). Previous studies have shown that the eyelet structure of healthy basal membrane ensures nutrient exchange between them. Damage of this normal structure results in epithelial basal cell migration, impeded differentiation, and epithelial or nutritional neuron diseases. After soaking the tissue in alcohol, the highly reflective granule-like materials and stroma structural change may decrease permeability in the basal membrane and hinder regeneration of the epithelium and sub-basal nerve plexus. The loose highly reflective granule-like debris we observed did not affect regeneration of the epithelium and sub-basal nerve plexus, which suggests that this material hindered regeneration of the epithelium and nerve plexus by damaging the normal basal membrane structure and function (Figure7)

Our conclusions were based on the abnormal epithelial region in corneal tissue visualized by confocal microscopy and lacks histopathologic confirmation. In a future study, we would prove our inference using a model animal to perform pathological examination on the discarded flap tissue in LASEK.

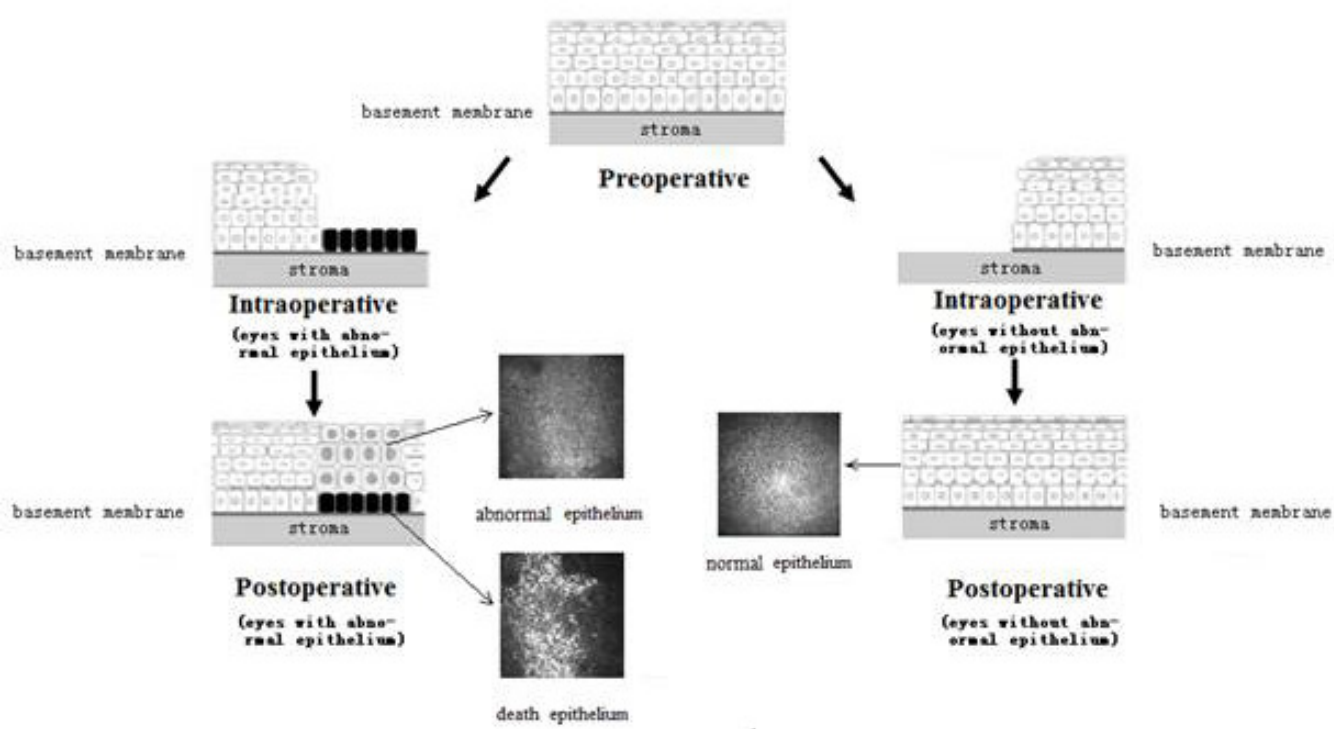

Figure 7. Abnormal area of epithelia cells might be caused by long-term reside of dead basal cells on the surface of epithalial basement memebrane and abnormal neurotrophic metabolism of cornea epithelia.

\section{Conflicts of interest}

The authors declare no conflict of interest.

\section{ACKNOWLEDGMENTS}

Research supported by grants from the Natural Science Foundation of China (\#81173306). 


\section{REFERENCES}

Bernátová V and Synek S (2011). Long term results of laser refractive operations-methods Epi-LASIK or LASEK. Cesk. Slov. Oftalmol. 67: 119-128.

Chan JJ, Farmer PJ, Southwell BR, Sourial M, et al. (2009). Calcitonin gene-related peptide is a survival factor, inhibiting apoptosis in neonatal rat gubernaculum in vitro. J. Pediatr. Surg. 44: 1497-1501.

Choi CY, Kim JY, Kim MJ, Tchah H, et al. (2008). Transmission electron microscopy study of corneal epithelial flaps following removal using mechanical scraping, alcohol, and epikeratome techniques. J. Refract. Surg. 24: 667-670.

Huang B, Fu H, Yang M, Fang F, et al. (2009). Neuropeptide substance P attenuates hyperoxia-induced oxidative stress injury in type II alveolar epithelial cells via suppressing the activation of JNK pathway. Lung 187: 421-426.

Kishi S, Shimoke K, Nakatani Y, Shimada T, et al. (2010). Nerve growth factor attenuates 2-deoxy-d-glucose-triggered endoplasmic reticulum stress-mediated apoptosis via enhanced expression of GRP78. Neurosci. Res. 66: 14-21.

Lee SJ, Kim JK, Seo KY, Kim EK, et al. (2006). Comparison of corneal nerve regeneration and sensitivity between LASIK and laser epithelial keratomileusis (LASEK). Am. J. Ophthalmol. 141: 1009-1015.

Lin N, Yee SB, Mitra S, Chuang AZ, et al. (2004). Prediction of corneal haze using an ablation depth/corneal thickness ratio after laser epithelial keratomileusis. J. Refract. Surg. 20: 797-802.

Long Q, Chu R, Zhou X, Dai J, et al. (2006). Correlation between TGF-beta1 in tears and corneal haze following LASEK and epi-LASIK. J. Refract. Surg. 22: 708-712.

Nakamura K, Kurosaka D, Bissen-Miyajima H and K. Tsubota. (2001). Intact corneal epithelium is essential for the prevention of stromal haze after laser assisted in situ keratomileusis. Br. J. Ophthalmol. 85: 209-213.

Pallikaris IG, Naoumidi Ò, Kalyvianaki MI, Katsanevaki VJ, et al. (2003). EpiLASIK: comparative histological evaluation of mechanical and alcohol assisted epithelial separation. J. Cataract. Refract. Surg. 29: 1496-1501.

Qazi MA, Sanderson JP, Mahmoud AM, Yoon EY, et al. (2009). Postoperative changes in intraocular pressure and corneal biomechanical metrics Laser in situ keratomileusis versus laser-assisted subepithelial keratectomy. J. Cataract. Refract. Surg. 35: 1774-1788.

Rajan MS, Watters W, Patmore A and Marshall J, (2005). In vitro human corneal model to investigate stromal epithelial interaction s following refractive surgery. J. Cataract. Refract. Surg. 31: 1789-1801.

Reilly CD, Panday V, Lazos V and Mittelstaedt BR (2010). PRK vs LASEK vs Epi-LASIK: a comparison of corneal haze, postoperative pain, and visual recovery in moderate to high myopia. Nepal. J. Ophthalmol. 2: 97-104.

Scerrati E (2001). Laser in situ keratomileusis $v$ s laser epithelial keratomileusis (LASIK vs LASEK). J. Refract. Surg. 17 (2 Suppl): S219-S221.

Taneri S, Feit R and Azar DT (2004). Safety, efficacy, and stability indices of LASEK correction in moderate myopia and astigmatism. J. Cataract. Refract. Surg. 30: 2130-2137.

Taneri S, Zieske JD and Azar DT (2005). Evolution, techniques, clinical outcomes, and pathophysiology of LASEK: review of the literature. Surv. Ophthalmol. 50: 502-504.

Taneri S, Oehler S, Koch J and Azar D (2011). Effect of repositioning or discarding the epithelial flap in laser-assisted subepithelial keratectomy and epithelial laser in situ keratomileusis. J. Cataract. Refract. Surg. 37: 1832-1846.

Thornton I, Puri A, Xu M and Krueger RR (2007). Low-dose mitomycin C as a prophylaxis for corneal haze in myopic surface ablation. Am. J. Ophthalmol. 144: 673-681.

You L, Kruse FE and Volcker HE (2000). Neurotrophic factors in the human cornea. Invest. Ophthalmol. Vis. Sci. 41: 692-702.

Yulish M, Beiran I, Miller B and Pikkel J (2012). Ascorbate prophylaxis with mitomycin-C for corneal haze after laserassisted sub-epithelial keratectomy. Isr. Med. Assoc. J. 14: 382-385. 